\title{
Photoacoustic microscopy of cerebral blood-oxygenation dynamics in mice
}

Konstantin Maslov, Erich W. Stein, Lihong V. Wang

Konstantin Maslov, Erich W. Stein, Lihong V. Wang, "Photoacoustic microscopy of cerebral blood-oxygenation dynamics in mice," Proc. SPIE 7177, Photons Plus Ultrasound: Imaging and Sensing 2009, 71770C (24 February 2009); doi: 10.1117/12.810036

SPIE. Event: SPIE BiOS, 2009, San Jose, California, United States 


\title{
Photoacoustic microscopy of cerebral blood-oxygenation dynamics in mice
}

\author{
Konstantin Maslov, Erich W. Stein, Lihong V. Wang \\ Optical Imaging Laboratory, Department of Biomedical Engineering, \\ Washington University in St. Louis, St. Louis, Missouri 63130
}

\begin{abstract}
In this work, we exploit the high depth and temporal resolutions of PAM to noninvasively image the blood-oxygenation dynamics of multiple cortex vessels in the mouse brain simultaneously in response to controlled hypoxic and hyperoxic challenges. The dark-field photoacoustic microscopy (PAM) technique was enhanced to image the cortex vasculature of the mouse brain in vivo using endogenous hemoglobin contrast with one second temporal resolution. The maximum values of about $20 \%$ with standard deviation $\pm 1.2 \%$ were found to vary significantly among the cortex vessels studied. The hypoxic response time to rise from $10 \%$ to $90 \%$ of maximum was $63 \pm 6 \mathrm{sec}$. The reverse response time for this event was $16 \pm 2$ sec.
\end{abstract}

Keywords: Photoacoustic imaging, photoacoustic microscopy, functional brain imaging, neuroimaging, noninvasive imaging, somatosensory cortex.

\section{INTRODUCTION}

In recent years, genetically altered mouse models have been developed to mimic neurological diseases of humans. These animal models permit the controlled study of the cause, effect, and treatment of neurological diseases, and are poised to provide clinically translatable insights. ${ }^{1}$ Hence, approaches to screen mouse brain function throughout disease progression are desired, making noninvasive techniques most appealing for longitudinal studies of the same subject. Since direct noninvasive techniques of locating and measuring brain activity (i.e., electrical signals) are not available, downstream surrogates for neuronal activity are used to noninvasively image brain activity. The most commonplace of which is the "hemodynamic response" - changes in blood oxygenation, volume and flow of local brain regions induced by neuronal activity. ${ }^{2}$

The distinctive spectral characteristics of hemoglobin make optical imaging techniques a good choice to monitor the hemodynamic response with high sensitivity. However, to provide noninvasive images of brain activity with high spatial resolution, these techniques must overcome the effects of light scattering from the scalp and skull. ${ }^{3}$ Photoacoustic (PA) imaging techniques are ideally suited for this application. ${ }^{4}$ PA techniques use short pulses of laser irradiation to induce thermoelastic expansion of biological tissues, which subsequently produces wideband ultrasonic waves (PA waves) that can be detected with an ultrasound receiver. Because PA wave generation can be induced by absorption of diffuse photons, and ultrasonic scattering in biological tissues is two orders of magnitude less than that of optical scattering, PA imaging techniques have been used to image structures deeper than one optical transport mean free path in tissue with a resolution in the tens of microns. ${ }^{5}$ Furthermore, since PA waves arise from absorbed optical energy distributions within tissues, PA image contrast is directly related to optical absorption. With hemoglobin being one of the dominant absorbers in tissues, PA imaging techniques are well equipped to image hemoglobin concentration and oxygenation in vascular networks in vivo by using multiple optical wavelengths. ${ }^{5}$

Other techniques such as $\mu \mathrm{PET}, \mu \mathrm{SPECT}, \mu \mathrm{CT}$ and fMRI have been successfully implemented to noninvasively image mouse brain structures in vivo. Functional brain imaging with $\mu \mathrm{PET}$ and $\mu \mathrm{SPECT}$, however, require exogenous contrasts and exhibit inherently low resolution (millimeter scale). ${ }^{6}$ fMRI is capable of imaging mouse brain structure and function by exploiting the endogenous differences in magnetic properties of oxy- and deoxyhemoglobin, ${ }^{7}$ but is unable to distinguish between increased blood oxygenation or decreased blood volume. ${ }^{3,8}$ PA tomography (PAT) was previously

Photons Plus Ultrasound: Imaging and Sensing 2009, edited by Alexander A. Oraevsky, Lihong V. Wang, Proc. of SPIE Vol. 7177, 71770C · @ 2009 SPIE · CCC code: 1605-7422/09/\$18 · doi: 10.1117/12.810036 
used to noninvasively image the cortex vasculature in the in vivo rat model and image neural activity by tracking local changes in cortical blood volume arising from somatosensory excitation; ${ }^{9}$ however, PAT in this implementation exhibits poor depth resolution. Additionally, the aforementioned techniques are reconstruction-based, requiring complete image acquisition to accurately measure single points of interest. Photoacoustic microscopy (PAM), however, operates in reflection-mode, and uses spherically focused ultrasonic detection to directly form images, affording direct measurements of regions of interest with notable depth and temporal resolution. ${ }^{5,10}$

Towards the goal of imaging brain activity in the mouse model, in this work the PAM technique ${ }^{5}$ was enhanced to noninvasively image the cortical vasculature of the mouse brain in vivo. Specifically, we increased the image acquisition rate and modified the ultrasonic detector to decrease image artifacts caused by ultrasonic reflections from the skull.

In this report, we track the real-time oxygenation dynamics of each of the selected multiple cortex vessels simultaneously in response to controlled perturbations of inhaled oxygen levels. These results demonstrate the ability of PAM to noninvasively assay the oxygen status of brain vessels and lay the groundwork for noninvasive imaging of brain activity via the hemodynamic response.

\section{METHODS AND MATERIALS}

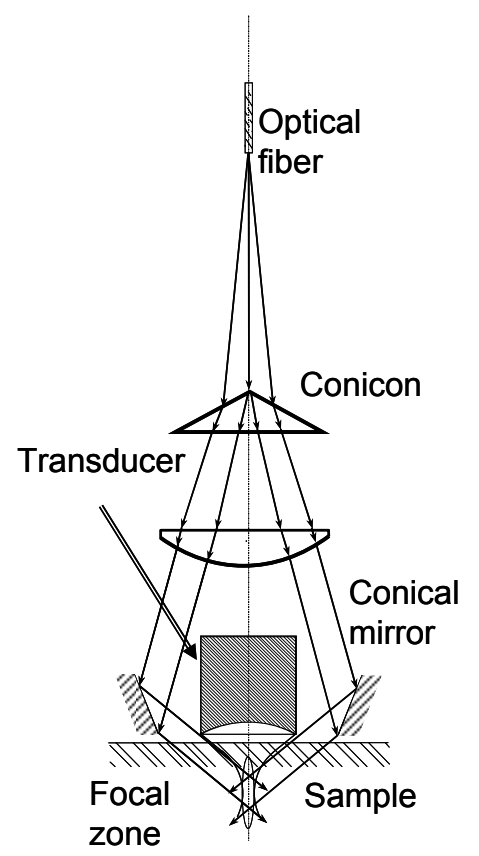

Fig. 1. Experimental setup.

To achieve the experimental goal, the photoacoustic system not only must be non invasive, but have good time resolution $(<1 \mathrm{~s})$, high sensitivity $(1 \%$ changes in $\mathrm{sO} 2)$, moderate to high resolution $(<100 \mathrm{~mm})$, and high contrast. The key components of our system are shown in Fig. 1. A laser pulse is delivered via optical fiber, expanded by a conical lens, reshaped by the convex lens, passed around the ultrasonic transducer, and focused by a conical mirror. The laser pulse along the ultrasonic transducer axis is confined to the transducer's depth of focus. The laser pulse penetrates through the surface of the tissue to a sufficient depth, selectively heating a volume of the tissue with higher optical absorption and producing ultrasonic waves which propagate with minimal alteration to the tissue surface. The ultrasonic waves are detected by an acoustic transducer, digitized, and transferred to a computer for data analysis. 
A Nd:YLF laser (INNOSLAB, Edgewave) pumped dye laser (CBR-D, Sirah) was used as the irradiation source. The laser pulse duration is $5 \mathrm{~ns}$ and the pulse repetition rate, which is controlled by the external trigger signal, was as high as $2 \mathrm{kHz}$. Laser output was coupled to an optical fiber, expanded through a conical lens and focused into the sample with an optical condenser. The resulting dark-field illumination pattern reduces optical fluence at the sample surface, partially averages superficial heterogeneities, and reduces superficial paraxial contributions. ${ }^{10}$ In this system, maximum optical fluence at $570 \mathrm{~nm}$ was measured to be $12 \mathrm{~mJ} / \mathrm{cm}^{2}$, less than the ANSI safety 1 limit ${ }^{11}$ for $400-700 \mathrm{~nm}\left(20 \mathrm{~mJ} / \mathrm{cm}^{2}\right)$. Laser-induced PA waves were detected by a focused ultrasonic transducer confocal with the optical lens. The PA signals detected by the ultrasonic transducer were amplified by $48 \mathrm{~dB}$ (ZFL 500LN, Mini-Circuits) before being digitized by a digital acquisition board (CompuScope 12400, Gage Applied). A motor driving PC controlled the scanner to perform raster scanning and sent trigger signals to both the data-acquisition PC and the pump laser. The trigger signals were synchronized with the clock-out signals from the digital acquisition board to minimize jitter.

The transducer is immersed in water inside a plastic container with an opening at the bottom that is sealed with a thin disposable polyethylene membrane. A window was opened at the bottom of the water tank and was sealed with an ultrasonically and optically transparent polyethylene membrane (thickness: $25 \mu \mathrm{m}$ ). An animal was placed under the water tank with the region of interest (ROI) exposed directly below the window. Ultrasonic gel (Clear Image, SonoTech) was applied to the ROI to achieve good acoustic coupling.

Images were formed by converting the recorded time-resolved PA signals into a one-dimensional depth-resolved image ("A-lines") along the $z$-axis using the sound velocity in soft tissue $(1.54 \mathrm{~mm} / \mu \mathrm{s})$. The rate of A-line acquisition is limited by the laser pulse repetition rate and mechanical scanning of the stage and was about $1 \mathrm{KHz}$ for the PAM used in this work. By raster scanning across the $x y$ plane, volumetric images of the sample were acquired at a rate one crosssectional image per second, which was fast enough to resolve most dynamic changes in blood oxygenation. For a detailed description of system components and operating principles, readers are referred to previous works. ${ }^{5,12}$

Swiss Webster mice (Harlan Sprague Dawley Inc., $\sim 25 \mathrm{~g}$ ) were selected as the animal models in this study. All in vivo procedures were carried out in conformity with the guidelines of the NIH and in compliance with the Washington University IACUC. The mouse was initially anesthetized using a cocktail ( $83 \mathrm{mg} / \mathrm{kg}$ Ketamine and $17 \mathrm{mg} / \mathrm{kg}$ Xylazine) administered by intraperitoneal (IP) injection. Body temperature was maintained at $37^{\circ} \mathrm{C}$ with a temperature controlled heating pad. Prior to imaging, the hair on the scalp was removed with an over-the-counter depilatory cream (Nair), as shown in Fig. 6c, and the animal was secured in a stereotaxic frame with a pair of ear pins and a tooth bar. Following hair removal and stereotaxic mounting, the anesthesia was transitioned to vaporized isoflurane $\left(1.5 \mathrm{~L} / \mathrm{min} \mathrm{O}_{2}, 0.75 \%\right.$ isoflurane) for the duration of all experimental procedures. Unlike previous studies ${ }^{13}$, no optical clearing agent was applied to the skin surface. After the completion of all experimental procedures, the animal was euthanized with a pentobarbital overdose administered via tail vein injection.

Using the setup previously described, ${ }^{5,12}$ a $50 \mathrm{MHz}$ ultrasonic detector (bandwidth: 70\%; NA: 0.44; focal length: 6.7 $\mathrm{mm}$; depth of focus: $0.3 \mathrm{~mm}$ ) and $570 \mathrm{~nm}$ illumination was used to image the cortical vasculature by raster scanning over the region of interest. However, a clear image of the cortical vasculature ( $>0.5 \mathrm{~mm}$ below the scalp surface) was not obtained, despite the ability to image structures $3 \mathrm{~mm}$ below the skin surface of the rat abdomen. ${ }^{10}$ Since the acquisition of PAM images is predicated upon optical energy delivery to excite PA waves and the subsequent collection of those PA waves via ultrasound detection, difficulties in imaging the cortical vasculature could arise from poor optical energy delivery or low acoustic energy collection. With this in mind, Monte Carlo simulations, ${ }^{13}$ using reported tissue optical properties, ${ }^{14}$ were used to compare the optical fluence at the brain surface to the optical fluence at $3 \mathrm{~mm}$ below the abdominal skin surface. Simulation results reveal that the optical fluence at the brain surface is approximately 17 times greater than the optical fluence at $3 \mathrm{~mm}$ below the abdominal skin surface, implying that the optical energy delivered to the cortical vessels on the brain surface was sufficient to generate PA waves. These results suggest that challenges in imaging the cortical vasculature arise from difficulties in collecting transcranial PA waves, which could be due to the acoustic impedance mismatch between the skull and surrounding soft tissues, as well as the acoustic attenuation of PA waves propagating through the skull. The most critical being the acoustic impedance mismatch between the skull and surrounding tissues, which could cause the pressure waves generated from the scalp to be reflected from the skull and be detected at a similar time as those originating from the cortical vasculature, thereby occluding the PA footprint of the cortical vessels as it is shown in Fig 3. Acoustic attenuation from the skull would curtail the PA waves generated from 
the cortical vessels, further complicating distinction. Correspondingly, experiments were conducted to examine how the skull affects the propagation of high frequency PA waves, with hopes of providing insight on optimizing the transcranial collection of PA waves generated from the brain vasculature.

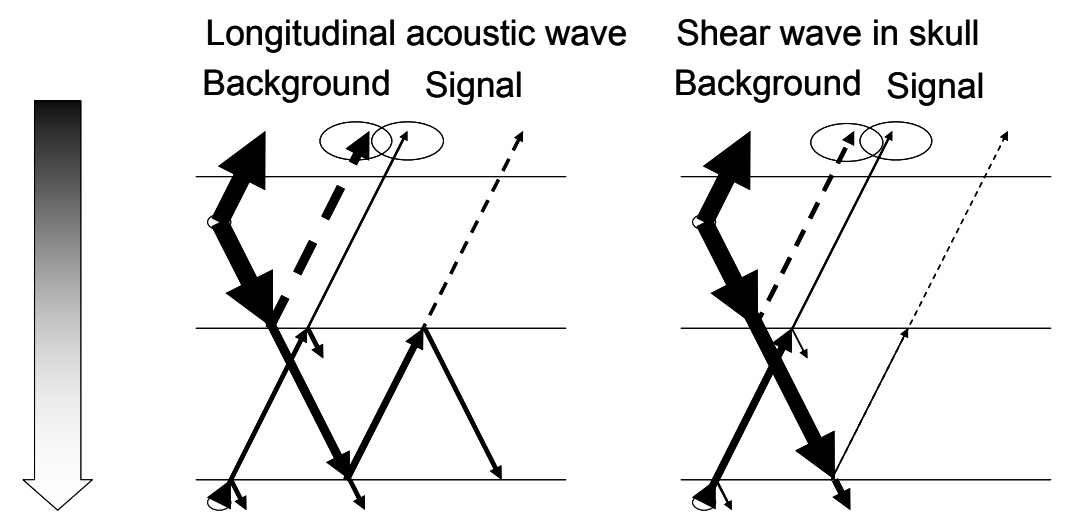

Fig. 3. Noisy background originated from optical absorption in scalp.

Due to size limitations of the mouse skull, the parietal bone of a freshly harvested rat skull was used as a surrogate. The parietal bone was immersed in water between two coaxially oriented unfocused ultrasonic transducers with $6 \mathrm{~mm}$ apertures. Ultrasound transmission through the parietal bone was measured at various incident angles and normalized to the system frequency response (Fig. 4). When holding the frequency constant, these data show decreased ultrasound transmission until a local minimum at $\sim 25$ degrees is observed. At incident angles above 25 degrees, the transmission of acoustic waves through the skull increases with incident angle. A similar trend has been shown by others, ${ }^{16}$ where incident acoustic waves beyond Snell's critical angle undergo a mode conversion from incident longitudinal waves to shear waves at the skull boundaries, then back into longitudinal waves at the interfacial layers (i.e. scalp or brain tissue).

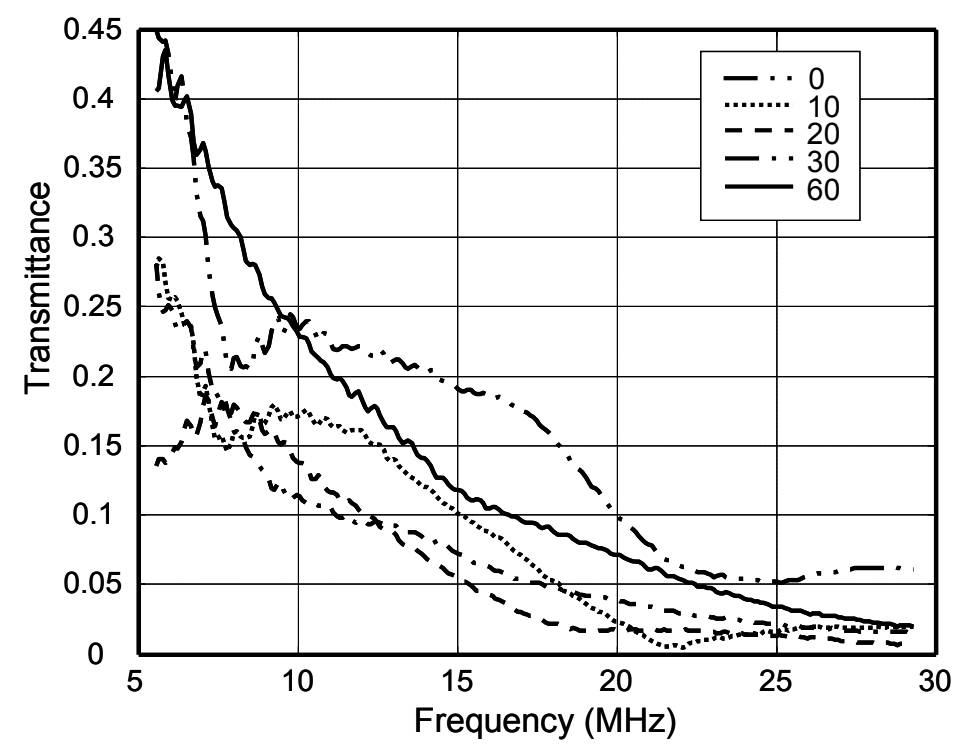

Fig. 4. Ultrasonic transmission through the rat skull at different incidence angles.

Additional calculations using known methods ${ }^{17}$ and skull acoustic parameters inverted from transmission measurements revealed that the skull reflection coefficient decreased by a factor of 6 at the largest measured incident angle compared to normal incidence due to the close acoustic impedances of shear waves in skull and $\mathrm{p}$ waves in soft tissue. Similar results were shown by others, ${ }^{18}$ suggesting that the reflection of PA waves from the skull and the reverberations within the skull 
become less prominent at high incident angles. Thus, it may be possible to eliminate reverberations by using annular ultrasonic detection. The design feasibility of such a detector is currently under investigation in our laboratory. In this report, we used a high NA acoustic lens, which collects more PA wave energy at oblique incidence angles. High NA detection would also compensate for acoustic energy losses from skull transmission by collecting more PA wave energy at the expense of reducing the focal length and depth of focus.

For a relatively large transducer PA signal amplitude can be found by integrating acoustic pressure, which includes propagating of the acoustic wave from transducer focal point through the skull (see Fig, 4). The resulting relative amplitude of the PA signal versus calculated transducer resolution $(0.72 \cdot \lambda / \mathrm{NA})^{-1}$ for ideal transducers of NA is shown in Fig. 5. According to the data shown in Fig. 5, a lower frequency bandwidth and larger aperture angle should allow more efficient collection of transcranial PA wave energy, leading to the design of an alternative detector for in vivo brain imaging. A $20 \mathrm{MHz}$ central frequency transducer with $91 \%$ nominal bandwidth was selected, and an acoustic lens was machined was into the silica delay line, yielding an NA of 0.64 , a focal length of $5 \mathrm{~mm}$. When compared to our previous detector (50 MHz, NA: 0.44), the $20 \mathrm{MHz}$ transducer has a significant frequency overlap with the transcranial acoustic waves and a 14 degree increase in the acceptance half-angle $(\theta=\arcsin (\mathrm{NA}))$, both of which should improve the collection efficiency of transcranial PA waves.

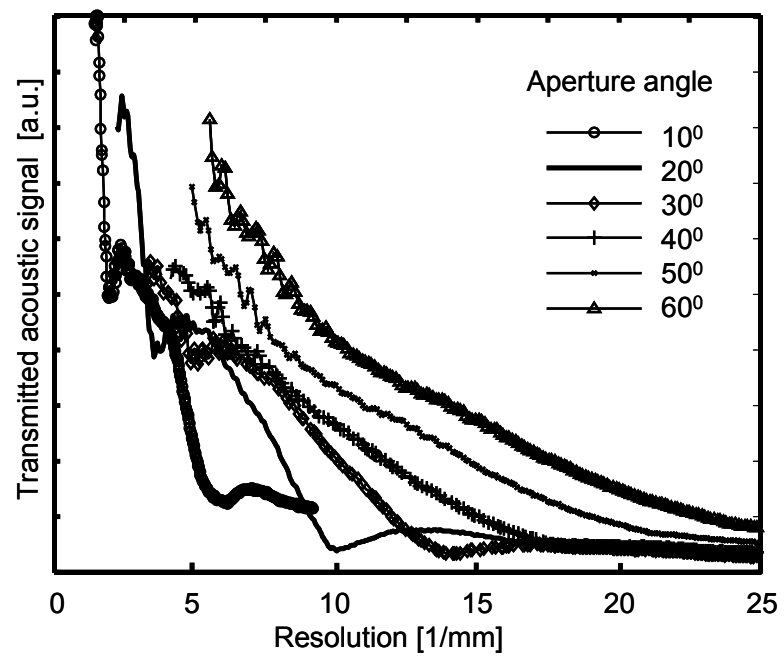

Fig. 5. PA signal amplitude of ideal transducers versus transducer resolution for different NA.

Since the resolving power of the PAM used in this work is related to the ultrasonic detection properties, namely bandwidth and NA, the lateral and axial resolutions were recharacterized using the $20 \mathrm{MHz}$ transducer prior to in vivo imaging. Specifically, the lateral resolution and depth of focus are determined by the ultrasonic transducer sensitivity to a point pressure source, similar in principle to those of pure optical imaging systems. ${ }^{19}$ The transducer sensitivity distribution can be approximated by the amplitude distribution in a converging spherical wave diffracted at a circular aperture. ${ }^{17}$ The axial resolution, however, is determined by the ability to resolve PA transients in the time domain and is inversely proportional to the transducer bandwidth. ${ }^{5}$ The lateral resolution was estimated by analyzing the PAM line spread functions (LSF) obtained by imaging a $6-\mu \mathrm{m}$-diameter carbon fiber. The lateral resolution was experimentally determined to be $70 \mu \mathrm{m}$ by taking the full width at half maximum (FWHM) of the lateral LSF. Our experimental data is similar to the estimated FWHM lateral resolution $r=0.72 \lambda_{0} / \mathrm{NA}=80 \mu \mathrm{m}$ at the center wavelength, $\lambda_{0}=v_{s} / f_{0}=71 \mu \mathrm{m}$, where $v_{s}$ is the approximate sound velocity in soft tissue $(1.5 \mathrm{~mm} / \mu \mathrm{s})$ and $f_{0}$ is the center frequency $(21 \mathrm{MHz})$ of the received PA signal.

Expected sensitivity (signal to noise ratio) of the PA system can be estimated by comparing Nyquist noise of transducer surface given by: 


$$
W_{n}=k T \Delta f n=1.38 \cdot 10^{-23} \cdot 300 \cdot 20 \cdot 10^{6} \cdot 2=16 \cdot 10^{-14} \mathrm{~W}
$$

to the power of the PA wave on transducer surface (including experimentally measured power losses of the transducer)

$$
W_{P A M}=\frac{p^{2}}{2 \rho v_{s}} K S=\frac{p^{2}}{2 \cdot 10^{3} \cdot 1.5 \cdot 10^{3}} \cdot 5.5 \cdot 10^{-3} \cdot 0.3 \cdot 10^{-4}=p^{2} \cdot 5.5 \cdot 10^{-14} \mathrm{~W},
$$

where: $k=1.38 \cdot 10^{-23}$ is Boltzmann constant , $T=300 \mathrm{~K}$ is temperature, $\Delta f$ is the FWHM (22 MHz) of the received PA signal frequency spectrum, $n \sim 2$ is noise figure, $\rho=10^{3}$ is mass density, $v_{s}$ is the approximate sound velocity in soft tissue $(1.5 \mathrm{~mm} / \mu \mathrm{s}), S=0.3 \cdot 10^{-4}$ is lens area, $K=5.5 \cdot 10^{-3}$ is transmission power losses, and $p$ is the he acoustic pressure generated by short laser pulse:

$$
p(r) \propto \frac{\beta c^{2}}{C_{p}} \mu_{a} H(r)=\mu_{a} \Gamma H(r) \sim 0.6 M P a .
$$

In (3) $\beta$ is thermal expansion coefficient, $C_{p}$ is specific heat, $\Gamma=0.11$ is Gruneisen parameter, $H=2 \cdot 10^{2} \mathrm{~W} / \mathrm{m}^{2}$ is optical fluence equal to safety limit and $\mu_{a} \sim 3 \cdot 10^{4} \mathrm{~m}^{-1}$ is blood optical absorption coefficient at $570 \mathrm{~nm}$. Using result of equation (3) in equation (2) and comparing equation (2) with equation (1) give SNR $>60 \mathrm{~dB}$. In practice SNR is significantly lower due to light and ultrasonic absorption amd ultrasonic reflection from the skull.

A conservative estimate of the axial resolution by taking the FWHM of the enveloped axial LSF yields an axial resolution of $54 \mu \mathrm{m}$. The enveloped axial LSF was obtained by taking the absolute value of the Hilbert transformed raw PA signal. The theoretical axial resolution estimated with $v_{s} / \Delta f$ is $67 \mathrm{um}$. These resolution values $-70 \mu \mathrm{m}$ lateral and $54 \mu \mathrm{m}$ axial - represent the ideal case; in in vivo situations, the resolution degrades owing to the frequency dependence of ultrasound absorption in the skull, which shifts the central frequency of the received PA signal from the central frequency of the ultrasonic transducer to $\sim 12 \mathrm{MHz}$. The estimated depth of focus $\Delta z=2 \lambda_{0} / \mathrm{NA}^{2}$ was 400 $\mu \mathrm{m} .{ }^{19}$

\section{NONINVASIVE IMAGING IN VIVO}

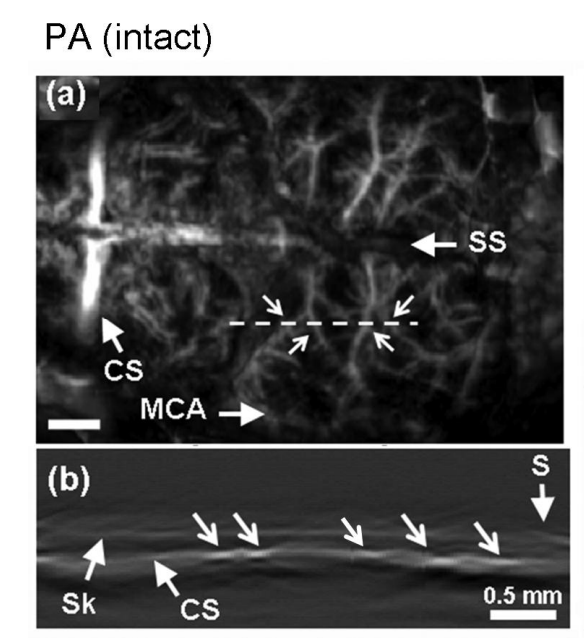

Optical (intact)

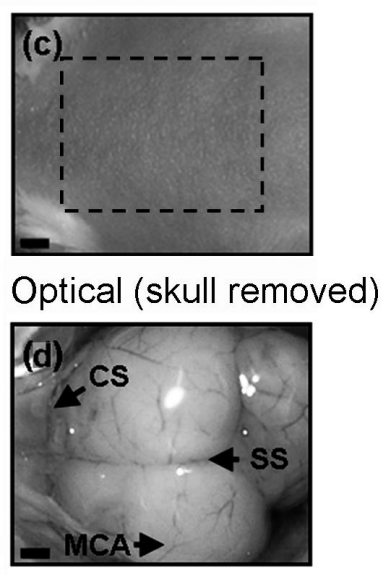

Fig. 6. In vivo image of the mouse cortex vasculature.

Using the $20 \mathrm{MHz}$ ultrasonic transducer and $570 \mathrm{~nm}$ illumination, PAM was used to noninvasively image the cortical vasculature of a mouse in vivo, by raster scanning over the region of interest (Fig. 6c). A clear image of the cortical vasculature was obtained with the scalp and skull intact (Fig.6a and b) in 7 minutes with step sizes of 20 and $40 \mu \mathrm{m}$ 
along $x$ and $y$, respectively. The maximum and mean contrast-to-noise ratio (CNR), defined as the intensity variation between the cortical vasculature and the background divided by the standard deviation of the background intensity, was 35 and $25 \pm 2 \mathrm{~dB}$, respectively. Signal gating was used to remove the PA signals originating from the scalp. Produced axial projection is displayed in 6a. Major vascular landmarks (sagittal sinus, middle cerebral artery and coronal suture, Fig.6a) were clearly identified and agree well with in situ anatomical photographs taken after image acquisition (Fig.6d). Furthermore, we emphasize a unique aspect of time-resolved PA imaging techniques: the ability to obtain twodimensional depth resolved images by line scanning. These images, often denoted as "B-scans," can be acquired in less than one second (acquisition time is currently limited by mechanical scanning) and also allow depth-dependent tissues such as the skin surface, skull, brain cortex surface and cortical vasculature to be clearly resolved. Finally, while the 20 $\mathrm{MHz}$ detector used in this work was engineered to image major vessels on the brain surface, we were successfully able to image an obliquely embedded black anodized pin $3.2 \mathrm{~mm}$ below the scalp surface.
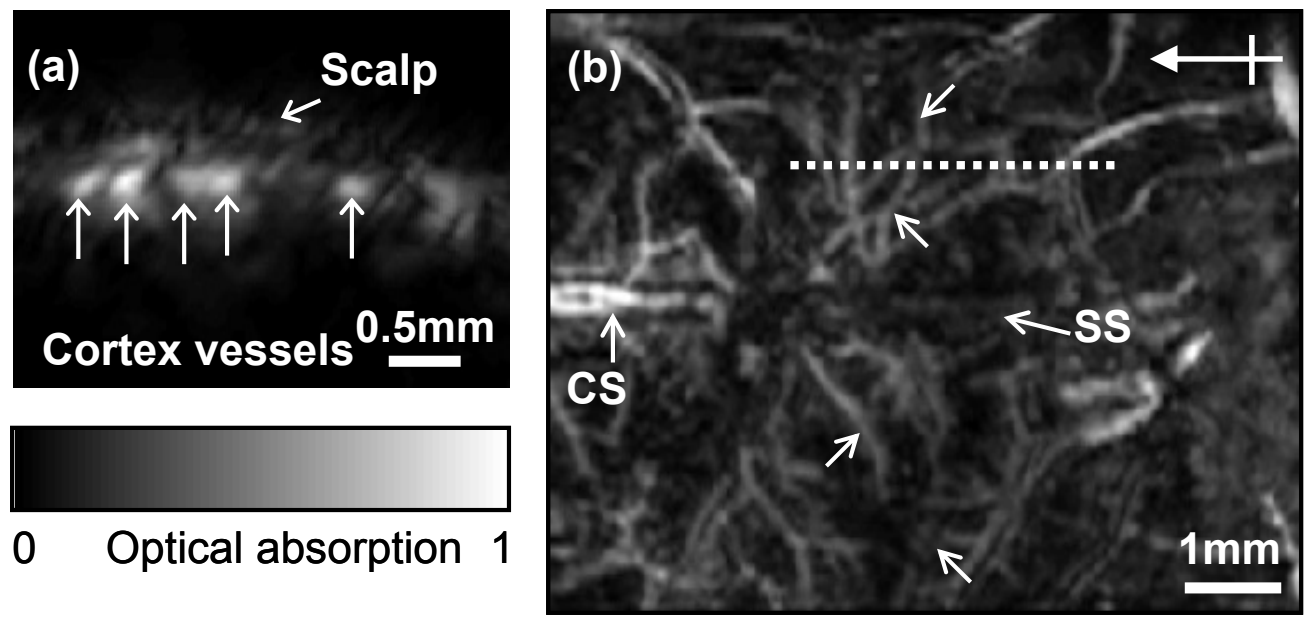

Fig. 7, In vivo image of the mouse cortex vasculature. (a) B-scan image obtained from scanning along the white dotted line in Fig. 1 b. Colored arrows indicate the cross sections of the cortex vessels whose dynamic response to hypoxia and hyperoxia were tracked and plotted in Fig. 8. (b) Noninvasive, PA image of the $7.50 \mathrm{~mm}$ x $6.25 \mathrm{~mm}$ region of mouse cortex vasculature. SS: sagittal sinus; CS: coronal suture. Unlabeled arrows indicate SC cortex feeding/draining vessels. Crossed arrow points in the rostral direction. Dotted white line indicates the area over which line scanning was performed.

Fig. 7 shows a noninvasive, in vivo image of the mouse brain cortex vasculature for the region of interest at $570 \mathrm{~nm}$, a hemoglobin isosbestic point, where optical absorption is independent of oxygenation status. This image was primarily used to aid in identifying the vasculature associated with the somatosensory cortex (SC) - a well defined model used to study the hemodynamic response. After identifying several major SC vessels, a cross section of the vessels was obtained by line scanning (Fig. 7a, dotted line). It is important to note that time-resolved PA imaging techniques can yield cross sectional depth resolved images by line scanning, which was acquired in less than one second.

After identifying the vessels of interest, functional imaging was performed using two optical wavelengths: $561 \mathrm{~nm}$, a deoxyhemoglobin dominant wavelength, and $570 \mathrm{~nm}$, an oxygen insensitive absorption wavelength of hemoglobin. A series of time-based images was obtained by acquiring sequential multiwavelength scans through continuous line scanning of the region indicated in Fig. 7b. The acquisition time for each multiwavelength scan (ten seconds) was mainly limited by the time required to tune the dye laser wavelength. Throughout image acquisition, hyperoxic and hypoxic states were induced by alternating the breathed oxygen levels delivered to the animal subject in the form of step changes between pure oxygen $\left(100 \% \mathrm{O}_{2}\right.$, to induce hyperoxia) and carbogen $\left(5 \% \mathrm{O}_{2}, 5 \% \mathrm{CO}_{2}\right.$, and $90 \% \mathrm{~N}_{2}$, to induce hypoxia) and vice versa. ${ }^{5}$ The animal was exposed to each step change in oxygen concentration for $\sim 6 \mathrm{~min}$, and a pulse oximeter was used to verify hyperoxic and hypoxic status. Experiments were performed with $100 \%$ and $5 \% \mathrm{O}_{2}$ baselines, respectively. Multiwavelength data were transformed into functional data through a ratiometric procedure commonly used to qualitatively track blood oxygenation in vivo. ${ }^{15}$ This procedure involved normalizing pixel-wise the image acquired at $561 \mathrm{~nm}$ (deoxyhemoglobin dominant) to the image acquired at $570 \mathrm{~nm}$, which served as an oxygeninsensitive reference. The end result was a time-series of functional images. Although quantitative $\mathrm{sO}_{2}$ measurements 
were also possible ${ }^{5,12,20}$, they require calibration for wavelength dependent tissue optical attenuation, ${ }^{5,20}$ which cannot be done minimally invasively in the brain.

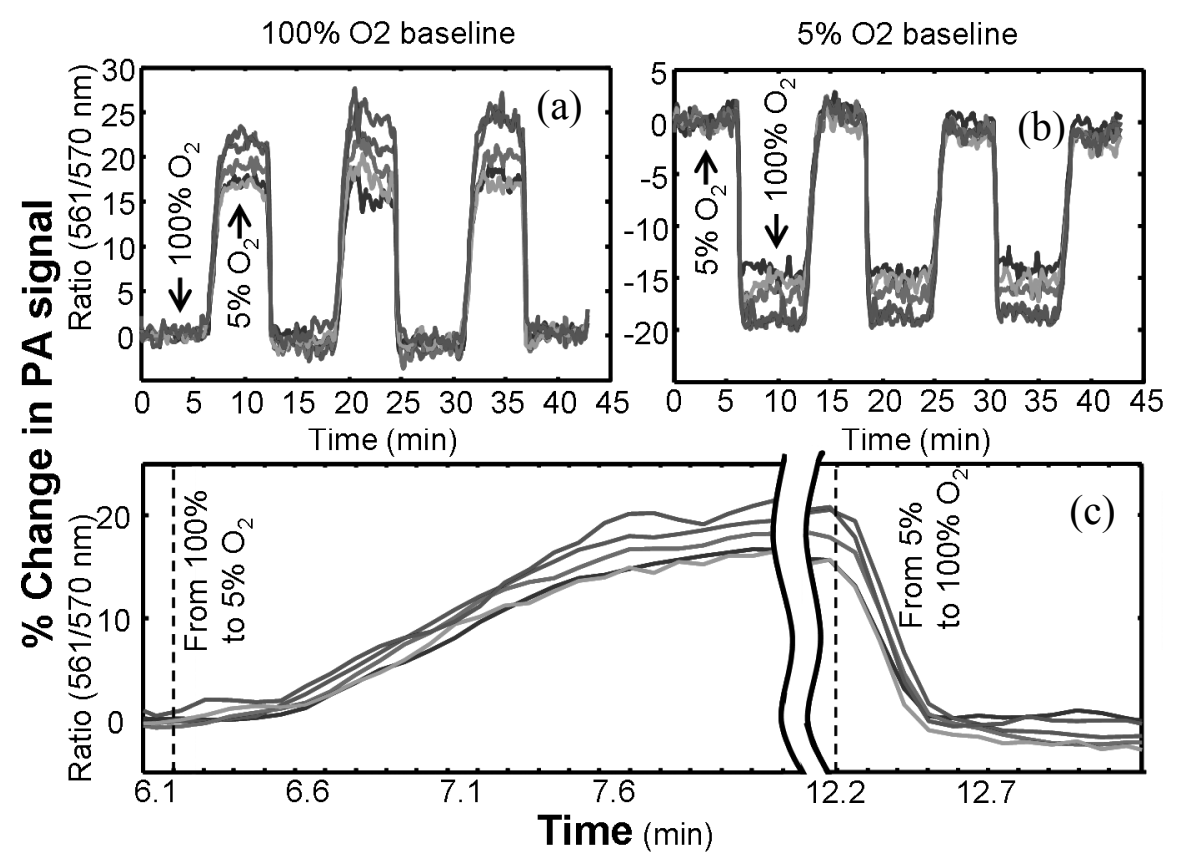

Fig. 8. Dynamic vessel response profiles. Profiles acquired through a hypoxic challenge and shown in percent change of ratiometric PA signals (a). Each trace corresponds to the response profile from the respective cortex vessel indicated with arrows in Fig 7a. Dynamic vessel response profile acquired through a hyperoxic challenge and shown in percent change (b). (c) Close-up of parts of the dynamic vessel responses shown in Fig. 8a.

Dynamic oxygenation profiles for each vessel shown in Fig. 7a (arrows), were obtained by integrating over the full width at half maximum (FWHM) of the image of each of the five vessels over the entire time-series. Pure $(100 \%) \mathrm{O}_{2}$ was used as a baseline, and the animal was subjected to three $5 \% \mathrm{O}_{2}$ steps with a return to baseline between successive hypoxic challenges. The resulting dynamic oxygenation profile for each of the five vessels is given in Fig. 8a. A closeup of the response curves corresponding to the forward and reverse response regions are shown in Fig. 2c to highlight the response transition phases. The initial step from hyperoxia $\left(100 \% \mathrm{O}_{2}\right)$ to hypoxia $\left(5 \% \mathrm{O}_{2}\right)$ occurred at time $t=6.1$ min. After an additional $27 \pm 3 \mathrm{sec}$. of lag time, a significant (two-fold greater than baseline fluctuations) increase in the ratiometric PA signal from each vessel was observed. The forward response time, characterized by the time required for the hypoxic response to rise from $10 \%$ to $90 \%$ of maximum, was $63 \pm 6 \mathrm{sec}$. We note that no significant variations $(\alpha=$ 0.1 ) in forward response time were observed for each of the five vessels studied. Interestingly, the maximum values of the hypoxic response values significantly varied among the cortex vessels studied, but no significant correlation between maximum response and vessel diameter was observed. At $12.2 \mathrm{~min}$., the hypoxic challenge was ended via a step exposure to $100 \% \mathrm{O}_{2}$, which decreased the levels of deoxyhemoglobin in the cortex vessels, causing a decrease in the ratiometric PA signal. The reverse response time for this event, defined as the time required for the hypoxic response to decrease from $90 \%$ to $10 \%$ of the maximum, was $16 \pm 2 \mathrm{sec}$. Our current system was not able to resolve any lag time associated with the transition from hypoxia to hyperoxia status. The repeated hypoxic challenges produced similar results in all cases.

A similar experiment was performed using $5 \% \mathrm{O}_{2}$ as a baseline (Fig 8b), The forward response time was determined to be $14 \pm 2$ sec., and the reverse response time was $(61 \pm 4 \mathrm{sec}$.), both of which agree well with the above experimental results. Furthermore, a similar lag time $(28 \pm 2$ seconds) was observed between the transition from hyperoxia and hypoxia. 


\section{Isosbestic point $(570 \mathrm{~nm})$}
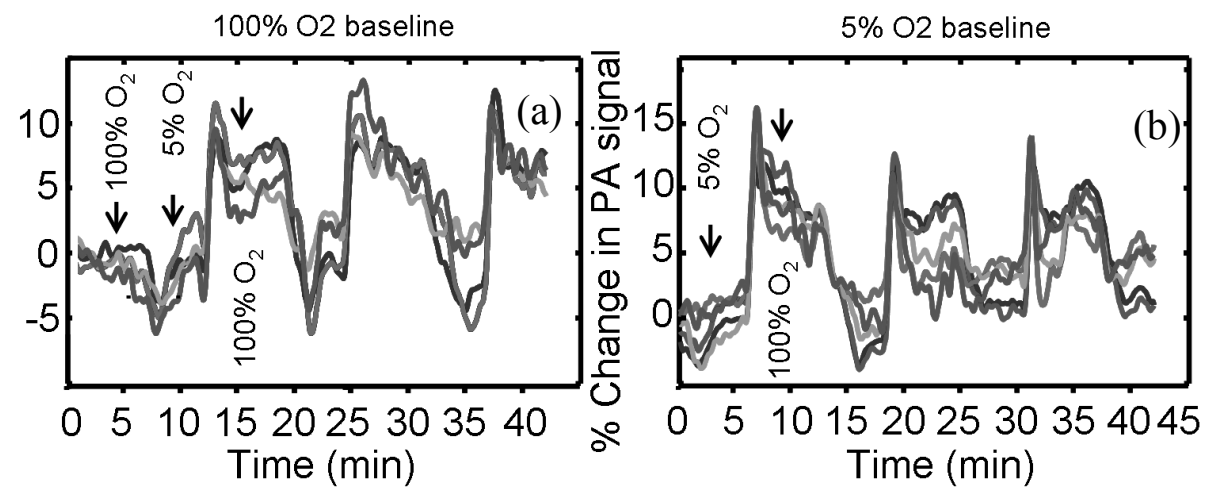

Fig. 9. Dynamic vessel response profiles. (a) Profiles acquired through a hypoxic challenge at isosbestic point (570 nm). Each trace corresponds to the response profile from the respective cortex vessel indicated with arrow in Fig 7a. (b) Dynamic vessel response profile acquired through a hyperoxic challenge at isosbestic point.

Dynamic vessel response profiles, measured at isisbestic point for each vessel shown in Fig. 7a, are shown in Fig. 9. To our surprise, Fig. 9 appears to show that switching to the hyperoxic state elicits an increase in the PA signal, contrary to an expected decrease in blood flow during hyperoxia ${ }^{21}$. This contradiction can be possibly explained as follows. The measured PA signal is proportional to both the light fluence on the vessel surface and the optical absorption coefficient in blood while weakly depending only on the vessel diameter ${ }^{20}$. Here, we imaged only relatively large diameter vessels. However, the optical attenuation in the skin, dura matter, and brain cortex strongly depends on the intervening volume of blood in much smaller vessels, where vasoconstriction is much higher. Correspondingly, a change in local fluence can overshadow a change of diameter or in hemoglobin concentration in the imaged vessel. The same effect can adversely affect the double wavelength measurement of oxygenation. However, to change the experimental result qualitatively, optical absorption in blood at $561 \mathrm{~nm}$ would have to be higher than that at $570 \mathrm{~nm}$, which corresponds to a physiologically unreasonable blood oxygenation level of less than $40 \%$ in the hyperoxic state. That is illustrated in Fig.10. Possible values of blood absorption in hyperoxic status are between two solid lines in Fig.10 which indicate oxyhemoglobin and deoxyhemoglobin optical absorption. Blood absorption at isosbestic point cannot change with change in blood oxygenation ( $\mathrm{sO} 2$ ) hence a $10 \%$ increase of the PA signal at hypoxia is caused by a $10 \%$ decrease of optical fluence. If we suppose that blood $\mathrm{sO} 2$ does not change at hypoxia and tissue scattering changes very little due to small shift of optical wavelength, possible values of blood absorption mast be within interval indicated by wide vertical arrow in Fig. 10. The experimentally measured value was above this interval, indicating a sO2 change towards deoxygenated hemoglobin, which confirms that the PA measurements correctly reflect change in blood oxygenation at least qualitatively.

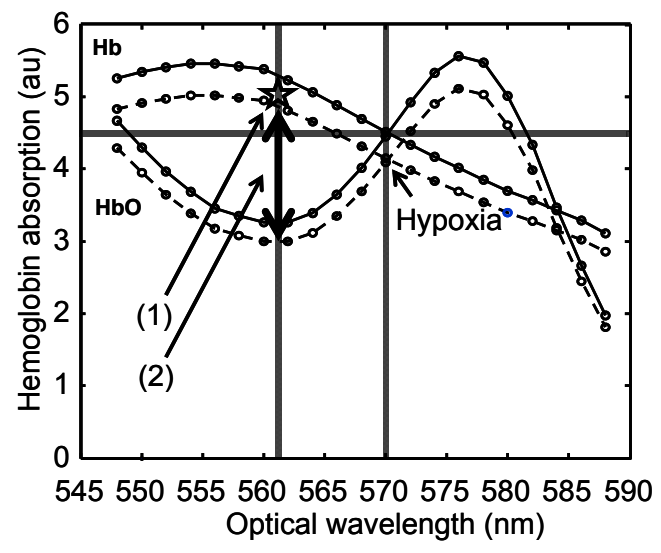

Fig. 10. Hemoglobin optical absorption at $570 \mathrm{~nm}$ and $561 \mathrm{~nm}$. (1) experimentally found blood absorption (2) interval of possible value of blood absorption for $\mathrm{sO} 2$ changing from $0 \%$ to $100 \%$. 
Results of the present work demonstrate the ability of PAM to track the oxygenation dynamics of each of multiple cortex vessels simultaneously in vivo. Furthermore, these data were also used to estimate the baseline standard deviation $( \pm 1.2 \%)$, which can be used to predict the detection limit of the current system. The limit of detection (LOD) is empirically defined as 3 times the standard deviation $\sigma$ of the baseline signal $( \pm 1.2 \%)$. Thus, LOD $=3 \sigma=3.6 \%$. We also note that a high degree of correlation $(0.89 \pm 0.2)$ was observed between the dynamic oxygenation profiles and the oxygen step change profiles (Figs. 8a and b).

In summary, we have successfully demonstrated the ability of PAM to noninvasively track the oxygenation dynamics of multiple cortex vessels in vivo by using hyperoxic and hypoxic challenges. The physiological responses observed under the hyperoxic and hypoxic challenges used in this work show the potential for PAM to be used to noninvasively monitor mouse brain activity via the hemodynamic response. The realization of this potential could provide a simple means to monitor the longitudinal effects of human-like neurological disease on brain function via transgenic mouse models.

\section{ACKNOWLEDGEMENT}

This project is sponsored in part by National Institutes of Health Grant Nos. R01 EB000712, R01 NS46214 (BRP), R01 EB008085 and U54 CA136398 (Network for Translational Research). L.W. has a financial interest in Endra, Inc., which, however, did not support this work.

\section{REFERENCES}

[1] M. Hafezparast, A. Ahmad-Annuar, N. W. Wood, S. J. Tabrizi and E. M. C. Fisher, "Mouse models for neurological disease," The Lancet Neurology 1 (4), 215-224 (2002).

[2] A. Grinvald, E. Lieke, R. D. Frostig, C. D. Gilbert and T. N. Wiesel, "Functional architecture of cortex revealed by optical imaging of intrinsic signals," Nature 324 (6095), 361-364 (1986).

[3] E. M. C. Hillman, "Optical brain imaging in vivo: techniques and applications from animal to man," Journal of biomedical optics 12 (5), 051402 (2007).

[4] M. H. Xu and L. H. V. Wang, "Photoacoustic imaging in biomedicine," Review of Scientific Instruments 77 (4), 041101 (2006).

[5] H. F. Zhang, K. Maslov, G. Stoica and L. V. Wang, "Functional photoacoustic microscopy for high-resolution and noninvasive in vivo imaging," Nat Biotechnol 24 (7), 848-851 (2006).

[6] R. S. Balaban and V. A. Hampshire, "Challenges in small animal noninvasive imaging," ILAR journal / National Research Council, Institute of Laboratory Animal Resources 42 (3), 248-262 (2001).

[7] G. Nair and T. Q. Duong, "Echo-planar BOLD fMRI of mice on a narrow-bore 9.4 T magnet," Magnetic Resonance in Medicine 52 (2), 430-434 (2004).

[8] E. T. Ahrens and D. J. Dubowitz, "Peripheral somatosensory fMRI in mouse at 11.7 T," Nmr in Biomedicine 14 (5), 318-324 (2001).

[9] X. D. Wang, Y. J. Pang, G. Ku, X. Y. Xie, G. Stoica and L. H. V. Wang, "Noninvasive laser-induced photoacoustic tomography for structural and functional in vivo imaging of the brain," Nature Biotechnology 21 (7), 803-806 (2003).

[10] K. Maslov, G. Stoica and L. V. H. Wang, "In vivo dark-field reflection-mode photoacoustic microscopy," Optics Letters 30 (6), 625-627 (2005).

[11] SPIE [American national standard for the safe use of lasers Z136.1], American National Standards Institute, New York, (2000).

[12] H. F. Zhang, K. Maslov and L. V. Wang, "In vivo imaging of subcutaneous structures using functional photoacoustic microscopy," Nat. Protocols 2 (4), 797-804 (2007).

[13] V. P. Zharov, E. I. Galanzha, E. V. Shashkov, N. G. Khlebtsov, and V. V. Tuchin, "In vivo photoacoustic flow cytometry for monitor of circulating single cancer cells and contrast agents," Optics Letters 31, 3623-3625 (2006).

[14] L. V. Wang, S. L. Jacques, and L.-Q. Zheng, "MCML - Monte Carlo modeling of photon transport in multi-layered tissues," Computer Methods and Programs in Biomedicine 47, 131-146 (1995). 
[15] V. Tuchin, [Tissue Optics: Light Scattering Methods and Instruments for Medical Diagnosis, Second ed.] SPIE, (2007).

[16] G. T. Clement, P. J. White and K. Hynynen, "Enhanced ultrasound transmission through the human skull using shear mode conversion," Journal of the Acoustical Society of America 115(3), 1356-1364 (2004).

[17] Maslov K., Kim R. Y, Kinra V. K, Pagano N. J. "A new technique for the ultrasonic detection of internal transverse cacks in carbon-fibre/bismaleimide composite laminates", Composites Science and Technology, 60(12) 2185-2190 (2000).

[18] P. J. White, G. T. Clement and K. Hynynen, "Longitudinal and shear mode ultrasound propagation in human skull bone," Ultrasound in Medicine and Biology 32(7), 1085-1096 (2006).

[19] M. Born and E. Wolf, [Principles of Optics, Sixth ed.] Pergamon, Cambridge, (2006).

[20] M. Sivaramakrishnan, K. Maslov, H.F. Zhang, G. Stoica and L.V. Wang "Limitations of quantitative photoacoustic measurements of blood oxygenation in small vessels," Phys. Med. Biol. 52, 1349-1361 (2007))

[21] Parthasarathi, K. \& Lipowsky, H.H. "Capillary recruitment in response to tissue hypoxia and its dependence on red blood cell deformability”. The American journal of physiology 277, 2145-2157 (1999) 